\title{
Soberanía e igualdad en el derecho internacional
}

\author{
Carlos D. Espósito*
}

\section{INTRODUCCIÓN}

Desde un punto de vista jurídico e internacional, la soberanía es un concepto que sirve para determinar cuáles son las libertades, prerrogativas y competencias de los Estados, pero también sus responsabilidades, que en la sociedad internacional se definen y encuentran sus límites en las reglas de Derecho internacional. Esa es la esencia de la famosa idea que la Corte Permanente de Justicia Internacional expresó en el caso Wimbledon ${ }^{1}$ en 1923, donde hubo de enfrentarse al difícil

Catedrático de Derecho Internacional Público, UAM. Agradezco a Nicolás CARRILLO SANTARELLI, becario de investigación en la UAM, su ayuda en la búsqueda de materiales sobre el tema de este trabajo. Este trabajo ha sido publicado en el Anuario de la Facultad de Derecho de la Universidad Autónoma de Madrid correspondiente a las jornadas sobre «Desafíos de la igualdad, desafíos a la igualdad», dirigidas por los profesores Alfonso RUÍZ problema de interpretar una norma del Tratado de Versalles que prescribía una limitación a la soberanía del Estado alemán ${ }^{2}$. Esa idea de soberanía estatal como un conjunto de competencias desagregadas ha sido de gran utilidad para explicar un Derecho que rige principalmente las relaciones entre Estados soberanos y formalmente iguales. Y es este el sentido en que la igualdad soberana de los Estados es un principio fundamental del Derecho internacional ${ }^{3}$, del que derivan MIGUEL y Andrea MACÍA, de 12 a 14 de noviembre de 2008.

1 Caso del S.S. Wimbledon, Permanent Court of International Justice, Series A, núm. 1, 1923.

2 Sobre el dilema de la soberanía en el Derecho internacional a través de un revisión del caso del Wimbledon, véase J. KLABBERS, «Clinching the Concept of Sovereignty: Wimbledon Redux", Austrian Review of International and European Law, núm. 3, 1998, pp. 345 ss.

3 Véase, por ejemplo, A. REMIRO BRO- 
otros tantos principios fundamentales de este ordenamiento jurídico, como la prohibición de intervención en los asuntos internos de los Estados o la inmunidad de los Estados en los tribunales de otros Estados. Ahora bien, la evolución del derecho y la sociedad internacionales afectan esas libertades de los Estados socavando y poniendo en duda el axioma de la igualdad soberana de los Estados y su utilidad como ficción jurídica, un fenómeno que se ha expandido e incrementado en las últimas décadas como consecuencia de la interdependencia y la globalización ${ }^{4}$. Esos efectos se hacen

TÓNS, Derecho internacional público. Principios fundamentales, Tecnos, Madrid, 1982, pp. 81-84; y A. Cassese, International Law, Oxford University Press, Oxford, 2001, pp. 88-91. Para una descripción actual y detallada del principio véase la entrada redactada por J. KOKOTT, "States, Sovereign Equality», Max Planck Encyclopedia of Public International Law (MPEPIL), edición online de R. WOLFRUM, http://www.mpepil.com, visitada el 17 de noviembre de 2008.

4 Veáse C. IZQUIERDO SANS, «Algunas respuestas del Derecho internacional a la globalización", Anuario de la Facultad de Derecho de la UAM, vol. 9, 2005, pp. 151 ss. También P.M. DUPUY, «International Law: Torn Between Coexistence, Cooperation and Globalization", European Journal of International Law, vol. 9, 1998, pp. 278 ss. Desde el punto de vista de las relaciones internacionales, véase C. DEL ARENAL, «La nueva sociedad mundial y las nuevas realidades internacionales: un reto para la teoría y para la política», en Cursos de Derecho Internacional y Relaciones Internacionales de Vitoria-Gasteiz 2001, pp. 17 ss. notar en todos los aspectos del derecho internacional, incluyendo hechos sobresalientes como la aparición de nuevos sujetos y actores que cambian el escenario normativo de una manera esencial y traen consigo problemas de inclusión en los procesos normativos de creación y aplicación del derecho e importantes cuestiones de legitimidad.

En las líneas que siguen voy a introducir el concepto de igualdad soberana de los Estados como principio fundamental del derecho internacional y los problemas que hoy plantea su vigencia como "ficción constitutiva» de dicho ordenamiento jurídico. Después de una aproximación general, trataré de mostrar algunos retos y desafíos a la igualdad soberana de los Estados en relación con tres grandes ámbitos normativos del derecho internacional: la subjetividad, los procesos de creación de normas y las formas de aplicación. Los temas elegidos dentro de estos grandes ámbitos, cuyos límites sólo se justifican por razones didácticas, no tienen pretensiones de exhaustividad; al contrario, son temas ilustrativos y, aunque su elección responde en gran medida a preferencias de este autor, constituyen ejemplos donde el lector encontrará conflictos que afectan significativamente al principio de igualdad soberana de los Estados. La conclusión expresa un juicio sobre la justificación de la igualdad soberana como valor jurídico en tiempos de interdependencia y globalización. 


\section{LA IGUALDAD SOBERANA DE LOS EsTADOS COMO FUNDAMENTO DEL DERECHO INTERNACIONAL}

La igualdad soberana ${ }^{5}$ de los Estados es quizá el principio más fundamental entre los que forman el núcleo del Derecho internacional. Tan es así que algún autor califica la igualdad soberana de los Estados incluso como la norma fundamental ${ }^{6}$ del derecho internacional.

Para comprender mejor la dimensión de este principio conviene recordar su aparición normativa y doctrinal en la sociedad internacional. Hay un acuerdo bastante extendido en la doctrina de derecho internacional en que los postulados de la igualdad soberana de los Estados provienen de la organización normativa de las relaciones

5 Se ha escrito en abundancia sobre este principio como fundamento del Derecho internacional. Dos trabajos monográficos interesantes publicados con posterioridad a la Carta de Naciones Unidas son: B. BOUTROS-GHALI, «Le principe d'égalité des Etats et les organizations internationales", Recueil des Cours de la Académie de Droit International, 1961-II, pp. 2 ss.; y P. KOOIJMANS, The Doctrine of the Legal Equality of States: an Inquiry into the Foundations of International Law, Sijthoff, Leyden, 1964.

6 C. TOMUSCHAT, «International Law: Ensuring the Survival of Mankind on the Eve of a New Century. General Course on Public International Law», Recueil des Cours de la Académie de Droit International, vol. 281, 2001, pp. 13 ss., en la p. 161 utiliza la palabra kelseniana «Grundnorm» para referirse a la igualdad soberana. interestatales surgida de los Tratados que consagraron la Paz de Westfalia de $1648^{7}$. Aunque cierta autonomía religiosa de los principados y los reinos ya había sido reconocida en tratados anteriores $^{8}$, tras la finalización de la Guerra de los Treinta Años se desautorizan las relaciones internacionales basadas en ciertas categorías jerárquicas, sean religiosas o seculares, de coordinación o subordinación ${ }^{9}$, que hasta ese momento habían estado en manos del papado y el emperador ${ }^{10}$, que dieron paso a la igualdad soberana. Dicha igualdad se convirtió a partir de ese período en una ficción legal constitutiva del sistema jurídico surgido del

7 Los Tratados de Osnabrück y Münster fueron firmados y entraron en vigor el 15 de mayo y el 24 de octubre 24 de 1648 , respectivamente.

8 En efecto, podría afirmarse también que el respeto igualitario a los principados y reinos, basado en el principio cuius regio, eius religio, fue reconocido muchos años antes en la Paz de Augsburgo de 1555.

$9 \quad$ C. SCHREUER, «The Waning of Sovereign State: Towards a New Paradigm of International Law", European Journal of International Law, vol. 4, 1993, pp. 447 ss.

10 El poder entre los reinos y principados, no obstante, se seguía manifestando a través de alianzas que reflejaban el equilibrio de poderes. Sobre esta doctrina, MORGENTHAU, Politics Among Nations, A. A. Knopf, New York, 1948, especialmente Parte IV. Para un análisis actual sobre las múltiples formas en que los hegemones interactúan con el Derecho internacional, véase $\mathrm{N}$. KRISCH, «International Law in Times Of Hegemony: Unequal Power and the Shaping of the International Legal Order», European Journal of International Law, vol. 16, 2005, pp. 369 ss. 
orden westfaliano compuesto por Estados, un sistema en que ese principio resultaba útil y necesario para regular las relaciones internacionales entre Estados que no reconocían poderes superiores que pudiesen determinar sus obligaciones basadas en su propio consentimiento, conformadas en gran medida de nociones y elementos de igualdad, como ocurría, por ejemplo, con la reciprocidad en los tratados.

Estos acontecimientos fueron acompañados por desarrollos de derecho natural que definían a los Estados como iguales a semejanza de la igualdad entre los individuos. La comparación ya había sido avanzada en el siglo XVI por Francisco de Vitoria, la retomó Samuel von Puffendorf en el siglo XVII y encontrará su apogeo en los escritos de Emeric de Vattel un siglo después. En la doctrina de derecho internacional es famosa su idea de que un enano es tan hombre como un gigante, y que una pequeña república no es menos soberana que el reino más poderoso. Vattel infería de esa igualdad la necesaria consecuencia de que la calificación de algo como legal para una nación es igualmente legal para otra, y que lo injustificable en una, es igualmente injustificable en la otra ${ }^{11}$.

11 E. de VATTEL, Le droit des gens ou Principes de la Loi naturelle appliqués a la conduite et aux affaires des nations et des souverains, Londres, 1758, Preliminaires, párrafo 18 ( «Puisque les hommes font naturallement égaux, \& que leurs droits $\&$ leurs obligations font les mêmes, comme venant également de la Nature, les Nations composées d'hommes, \& consi-
La igualdad soberana, sin embargo, fue durante mucho tiempo una norma fundamental cuya aplicación se restringía a algunos Estados europeos. En otras palabras, la igualdad soberana no operaba fuera del contexto europeo, ya que solo los Estados europeos reunían las características necesarias para ser considerados iguales según ese derecho internacional, esto es, una cierta homogeneidad cultural y una comunidad de valores e intereses. La desigualdad que manifiesta este tratamiento, que diferenciaba a los civilizados de los bárbaros y los salvajes, ha supuesto una lucha importante a lo largo de la historia por expandir la aplicación del principio de igualdad y ha sido la causante de cier-

dérées comme autant de personnes libres qui vivent ensemble dans l'état de Nature, sont naturellement égales, \& tiennent de la Nature les mêmes obligations \& les mêmes droits. La puissance ou la faiblesse ne produisent, à cet égard, aucune différence. Un Nain est aussi bien un homme qu'un Géant: Un Petite République n'est pas moins un Etat souvarain que les plus puissant Roïaume»). Vattel era suizo y su defensa de la igualdad se explica por su creencia en el de Derecho natural, pero también se explica con apoyo en sus intereses nacionales, porque Suiza era una república pequeña frente a las grandes monarquías de su época y mediante la adopción del principio de igualdad soberana asumía a la vez la defensa de la prohibición de intervenir en los asuntos internos de los Estados. Sobre este tema, T. H. LEE, «International Law, International Relations Theory, and Preemptive War: the Vitality of Sovereign Equality Today", Law and Contemporary Problems, 2004, pp 151 ss. 
tos defectos de nacimiento del derecho internacional que aún hoy representan desafíos importantes para dicho ordenamiento normativo ${ }^{12}$. En efecto, esa «misión civilizadora» adjudicada al derecho internacional decimonónico ${ }^{13}$ ha servido para justificar la dominación de algunos Estados europeos sobre el resto del mundo, los diferentes, cuya soberanía era insuficiente en la medida en que no fuese europeizada u occidentalizada ${ }^{14}$. Este orden jurí-

12 Un ejemplo sobresaliente se encuentra en el artículo 38.1.c. del Estatuto de la Corte Internacional de Justicia, cuando establece que la Corte aplicará «los principios generales de derecho reconocidos por las naciones civilizadas» para solucionar las controversias que le sean sometidas. Sobre su significado jurídico, véase el comentario de A. PELLET al artículo 38 del Estatuto en A. ZIMMERMANN, C. TOMUSCHAT y K. OELLERS-FRAHM, The Statute of the International Court of Justice. A Commentary, Oxford University Press, Oxford, 2006, pp. 677 ss., esp. párrafos 245264.

13 M.KOSKENNIEMI, The Gentle Civilizer of Nations. The Rise and Fall of International Law 1870-1960, Cambridge University Press, Cambridge, 2004.

14 A. ANGHIE, Imperialism, Sovereignty and the Making of International Law, Cambridge University Press, Cambridge, 2004. Para este autor la misión civilizadora no termina con la colonización, sino que continúa a través de otros medios, como la clasificación de los Estados en desarrollados y subdesarrollados. Entre nosotros, el Profesor Antonio Remiro ha escrito un pequeño magnífico libro aplicando esas categorías decimonónicas al nuevo orden internacional proclamado a principios de la década de 1990: A. REMIRO BROTÓNS, Civilizados, bárbaros y salvajes en dico europeo que calificaba a los Estados según su grado de civilización y determinaba ciertas reglas a la luz de la condición de los entes involucrados, permitiendo por ejemplo las llamadas intervenciones "civilizadoras» o los «tratados desiguales» ${ }^{15}$, se manifiesta también de alguna manera en la actualidad, aunque con nombres distintos, cuando se tratan de motivar jurídicamente actos según categorías o clasificaciones que crean divisiones con el objeto de discriminar, como ocurre con los Estados canallas y los Estados fallidos ${ }^{16}$. Si bien la formulación de estas categorías no vulneraría el derecho internacional si se limitase a un plano de declaraciones políticas, su traducción en el menoscabo directo o indirecto de los derechos inherentes a la soberanía de los Estados puede ser indicio de su ilegalidad según el ordenamiento jurídico internacional. El efecto más claro de tal calificación sobre los derechos de los Estados sería la supresión del derecho a la no intervención en los asuntos internos del Estado fallido, pero también supondría la estigmatización de ciertos Estados como sujetos de segunda categoría, ante los

el nuevo orden internacional, McGrawHill, Madrid, 1997.

15 W. MORVAY, «Unequal Treaties», en R. Bernhardt (ed.) Encyclopedia of Public International Law, Vol. 7, North Holland, Amsterdam, 1984, pp. 514 ss.

16 P. MINNEROP, "The Classification of States and the Creation of Status within the International Community , Max Planck Yearbook of United Nations Law, vol. 7, 2003, pp. 79 ss. 
cuales podrían realizarse acciones no permitidas contra los iguales, como la legítima defensa preventiva contra los Estados canallas ${ }^{17} \mathrm{o}$ la falta de reconocimiento de la inmunidad de jurisdicción de loes Estados en relación con juicios por compensación de daños y perjuicios para los «Estados que patrocinan el terrorismo ${ }^{18}$. Por lo pronto, esta práctica denominativa no ha generado a su vez una práctica real en uno $\mathrm{u}$ otro sentido relevante para el derecho internacional, por ejemplo, ese tipo de calificaciones no ha afectado a la membresía de los Estados señalados en las organizaciones internacionales y la restricción de los derechos internacionales de algunos Estados llamados fallidos como consecuencia de su falta de capacidad para actuar con responsabilidad en la escena internacional ha sido siempre designada como excepcional ${ }^{19}$.

\footnotetext{
Ibid., pp. 158 ss.

18 Ibid., pp. 111 ss.

19 La piratería y los robos armados en el mar
} frente a las costas somalíes por parte de nacionales de Somalia han creado una situación de inseguridad que ha llevado al Consejo de Seguridad de las Naciones Unidas a adoptar una serie de resoluciones obligatorias que restringen los atributos soberanos de dicho Estado, si bien es cierto que todas las resoluciones se cuidan de manifestar que la situación es excepcional. Véase las resoluciones del Consejo de Seguridad S/RES/1816 (2008), S/ RES/1838 (2008), S/RES/1846 (2008) y S/ RES/1851(2008), todas disponibles en http://www.un.org/spanish/docs/sc08/ scrl08.htm. Sobre el tema, D. GUILFOYLE, "Piracy Off Somalia: UN Security Council Resolution 1816 and IMO Regio-
La consagración convencional del principio de igualdad soberana de los Estados llega con la Carta de las Naciones Unidas en un momento histórico en el que había tenido éxito el proceso de estatalización ${ }^{20}$, que extendió el modelo de organización política territorial de Estados occidentales por todo el mundo. En efecto, la Carta de Naciones Unidas dio origen a una nueva fase en la evolución de la igualdad soberana de los Estados no sólo porque declara que la propia Organización está basada en dicho principio, sino también porque introduce el objetivo de la descolonización, que permite la incorporación a la Organización de los nuevos Estados que accedían a la independencia y que asumían el principio de igualdad soberana en todos sus elementos como garantía del respeto de su soberanía interna, mediante reglas de no intervención e integridad territorial, y de su soberanía externa, a través de un reconocimiento de sus derechos en las relaciones con otros Estados.

La Carta de las Naciones Unidas establece en el primer párrafo del artículo 2 que «la Organización está basada en el principio de igualdad soberana de todos sus miembros» ${ }^{21}$. Este principio

nal Counter-Piracy Efforts», International and Comparative Law Quarterly, vol. 57, 2008, pp. 690 ss.

20 C. DEL ARENAL, supra nota 4, pp. 42-43.

21 A. BLECKMANN y B. FASSBENDER, «Article 2 (1)», en B. Simma (ed.), The Charter of the United Nations: A Commentary, vol. 1, Oxford University Press, Oxford, 2da edición 2002, pp. 68 ss.; M. 
es reiterado en la Declaración sobre los principios de Derecho Internacional referentes a las relaciones de amistad y a la cooperación entre los Estados de conformidad con la Carta de las Naciones Unidas $^{22}$, que tras proclamar que «Todos los Estados gozan de igualdad soberana», especifica que los Estados «tienen iguales derechos e iguales deberes y son por igual miembros de la comunidad internacional, pese a las diferencias de orden económico, social, político o de otra índole» ${ }^{23}$. El principio que contiene la Declaración es particularmente ilustrativo porque define el contenido de la igualdad soberana. En efecto, en la Declaración se dice que «en particular, la igualdad soberana comprende los elementos siguientes:

a) Los Estados son iguales jurídicamente; b) Cada Estado goza de los

KOHEN, «Article 2 Paragraph 1», en J.P. COT y A. PELLET (eds.), La Charte des Nations Unies: Commentaire Article par Article, vol. 1, Economica, Paris, 3ra edición 2005, pp. 399 ss.

22 Resolución 2625 (XXV), de 24 de octubre de 1970, de la Asamblea General de las Naciones Unidas.

23 Cf. la elegante formulación del principio que aparece en el artículo 10 de la Carta de la Organización de Estados Americanos, bajo el capítulo sobre los «derechos y los deberes fundamentales de los Estados»: «Los Estados son jurídicamente iguales, disfrutan de iguales derechos e igual capacidad para ejercerlos, y tienen iguales deberes. Los derechos de cada uno no dependen del poder de que disponga para asegurar su ejercicio, sino del simple hecho de su existencia como persona de derecho internacional». derechos inherentes a la plena soberanía; c) Cada Estado tiene el deber de respetar la personalidad de los demás Estados; d) La integridad territorial y la independencia política del Estado son inviolables; e) Cada Estado tiene el derecho a elegir y a llevar adelante libremente su sistema político, social, económico y cultural; f) Cada Estado tiene el deber de cumplir plenamente y de buena fe sus obligaciones internacionales y de vivir en paz con los demás Estados.»

Estos elementos de la igualdad soberana sobrepasan la mera idea de igualdad de los Estados, en la medida en que tratan de preservar su soberanía interna y externa, así como su independencia. Son unos elementos que definen un principio ideal de muy amplio alcan$\mathrm{ce}^{24}$, limitado únicamente por el deber de cumplimiento pleno y de buena fe del derecho internacional. Conviene subrayar esta limitación porque descansa sobre la base del imperio del derecho en la sociedad internacional. En efecto, los Estados son reconocidos como iguales y soberanos, pero no están por encima

24 En efecto, esa lista permite a los Estados argumentar sobre la base del principio de igualdad en infinidad de situaciones. Valga como ejemplo la defensa de Serbia respecto de la ilegalidad de la declaración unilateral de independencia de Kosovo, que Serbia calificó en el Consejo de Seguridad de las Naciones Unidas como una violación del principio de igualdad soberana de los Estados. Actas de la reunión 5839 del Consejo de Seguridad, de 18 de febrero de 2008, Documentos de Naciones Unidas S/PV.5839, p. 4. 
del derecho internacional. Esta es, por lo demás, la idea que refleja la decisión de la Corte Internacional de Justicia en el caso del Wimbledon donde se sostiene la libertad de los Estados sujeta al derecho internacional ${ }^{25}$. El hecho es que la evolución del derecho internacional y las profundas transformaciones de la sociedad internacional afectan a todos los elementos de la igualdad y suponen desafíos graves para el derecho internacional. A continuación, como anuncié en la introducción, trataré de ilustrar esos desafíos a la igualdad soberana desde las perspectivas de la subjetividad, las fuentes de producción del derecho internacional y su aplicación.

\section{III.LA IGUALDAD SOBERANA DESAFIADA}

\section{A. La igualdad jurídica de los Estados y la crisis de la subjetividad en el derecho internacional}

Resulta interesante destacar que la formulación de la soberanía como libertad del Estado limitada por el derecho internacional permite una evolución del concepto de igualdad soberana con excepciones nacidas del consentimiento de los Estados, del consenso de los Estados o incluso de obligaciones de derecho internacional surgidas más

25 Véase supra nota 1; y G. NOLTE, «Sovereignty as Responsibility", Proceedings of the Annual Meeting of the American Society of International Law, 2005, pp. 389 ss. allá del consentimiento de los Esta$\operatorname{dos}^{26}$. Esto es así porque la igualdad soberana se configura como una igualdad ante el derecho más que como una igualdad de derechos ${ }^{27}$. En sus orígenes, la noción de igualdad soberana de los Estados, quizá como consecuencia de la concepción antropológica de Vattel, era considerada más bien como un derecho fundamental del Estados. Hoy esa concepción está superada por los hechos y el derecho. La necesidad de cooperar hace cada vez más difícil o casi imposible mantener dominios reservados de soberanía inmaculados. $\mathrm{La}$ interdependencia socaba en muchos aspectos la idea de igualdad soberana. De todas formas, la igualdad soberana no se constituye como un derecho de los Estados sino como un principio general que admite excepciones consentidas o toleradas por los Estados, que en algunos casos resultan de la búsqueda de igualdad sustantiva entre los Estados y en otros reflejan la desigualdad material de los Estados. En esta parte pretendo exponer qué significa en realidad el elemento de la igualdad jurídica de los Estados para después mostrar algunas carencias del principio de igualdad soberana en la sociedad internacional de hoy.

En efecto, el primer elemento de la Declaración sobre los principios de

26 Véase C. TOMUSCHAT, «Obligations Arising for States Without or Against their Will», Recueil des Cours de l'Académie de Droit International, vol. 241-IV, 1993, pp. 195 ss.

27 J. KOKOTT, supra nota 3, párrafo 23. 
derecho internacional establece que los Estados son iguales jurídicamente. El significado de tal afirmación alude a la falta de jerarquías entre los Estados desde el punto de vista jurídico, a que los Estados no están subordinados unos a otros sin su consentimiento. Pero, en todo caso, se refiere más bien a un sentido formal de la igualdad. La igualdad material fue una preocupación doctrinal importante en las décadas de 1960 y 1970, durante las cuales se propuso la elaboración de un derecho internacional con normas diferenciadas según el nivel de desarrollo de los Estados. El derecho internacional del desarrollo pretendía darle contenido real y no simplemente formal a la regulación de las relaciones entre Estados soberanos pero con economías desiguales. Las propuestas del derecho internacional del desarrollo se tradujeron en una serie de resoluciones y documentos de Naciones Unidas impulsados por una mayoría de Estados en desarrollo que finalmente no se convirtieron en derecho internacional positivo y quedaron como un ideal normativo ${ }^{28}$. Salvo que aparezca una verdadera solidaridad internacional, la diferenciación normativa generalizada que pretendía el derecho internacional del desarrollo resulta hoy aún

28 Entre las publicaciones sobre el Derecho internacional del desarrollo, la que mejor expresa el ideal de la persecución de una justicia distributiva y material es el libro de M. BEDJAOUI, Hacia un Nuevo Orden Económico Internacional, UNESCO Editorial Sígueme, Salamanca, 1979. más complicada que entonces como consecuencia de la globalización ${ }^{29}$. No obstante, algunos tipos concretos de materialización de la justicia. No obstante, alguinos tipos contretos de materialización de la justicia con elementos distributivos podrían conseguirse «descendiendo de los principios primarios a los secundarios, de los estándares normativos abstractos a los concretos ${ }^{30}$. En este sentido, si se persigue la finalidad de una justicia distributiva hacia una igualdad sustantiva, creo que el énfasis se debería poner en el reforzamiento de los derechos humanos sociales y económicos, para hacer justicia social mediante el fortalecimiento de los derechos humanos ${ }^{31}$.

29 T. FLORY, «Mondialisation et droit international du développement», Revue générale de droit international public, vol. 101, 1997, pp. 609 ss. Este autor sostiene que en la globalización se ha perdido el hilo conductor del Derecho internacional del desarrollo, que estaba constituido por la distinción entre Estados que tenían derecho a un trato especial de acuerdo con un sistema jurídico diferenciado, caracterizado por una dualidad de normas. Sin embargo, deja la puerta abierta para desarrollos concretos, como el que podría producirse a través de un reforzamiento de la protección de los derechos humanos de carácter social o, según su expresión, de un Derecho internacional del desarrollo social.

30 A. REMIRO BROTÓNS, Derecho internacional público. Principios fundamentales, Madrid, Tecnos, 1982, p.

31 Véase D. BILCHITZ, Poverty and Fundamental Rights, Oxford University Press, Oxford, 2007. Véase también el excelente trabajo de M. IGLESIAS VILA, "Justicia global y derechos humanos: hacia una ética de las prioridades", Anuario de la 
La igualdad soberana de los Estados, en consecuencia, sigue teniendo un sentido formal más que sustantivo. Dicha igualdad soberana, como dije en la introducción, está en la base de derechos tradicionales de los Estados en la sociedad internacional, como la no intervención en los asuntos internos de otros Estados o la inmunidad de jurisdicción de los Estados en los tribunales de otros Estados. Sin embargo, tomando como ejemplos estos dos derechos estatales, se puede afirmar que aunque el núcleo de estos derechos siga vigente, ambos se encuentran sometidos a una gran presión que proviene de la aceptación de obligaciones internacionales de respeto y protección de derechos humanos, que incluyen derechos imperativos o ius cogens.

En efecto, en cuanto al principio de no intervención, por más que su contenido esencial siga siendo reconocido y garantizado por el derecho internacional ${ }^{32}$, está sometido en la

Facultad de Derecho de la UAM, vol. 9, 2005, pp. 41 ss.

32 Caso de las actividades militares y paramilitares en y contra Nicaragua (Nicaragua $v$ Estados Unidos de América), ICJ Reports 1986, p. 106, párrafo 202 (disponible en http://www.icj-cij.org/docket/files/70/6503.pdf):

«The principle of non-intervention involves the right of every sovereign State to conduct its affairs without outside interference; though examples of trespass against this principle are not infrequent, the Court considers that it is part and parcel of customary international law».

Un concepto que ha había sido afirmado en 1949 por la Corte Internacional de actualidad a desafíos derivados de la protección de los derechos humanos cuando su violación grave o sistemática no puede o no quiere ser evitada o cesada por el Estado donde ocurren estas atrocidades. Tragedias como las del genocidio en Rwanda han llevado a acentuar la idea de soberanía como responsabilidad y a tratar de afianzar un principio de responsabilidad de proteger $^{33}$. El principio aparece con esas características por vez primera en el informe de la Comisión Internacional sobre Intervención y Soberanía de los Estados ${ }^{34}$ y luego es adoptado

Justicia en el caso del Estrecho de Corfú, I.C.J. Reports 1949, p. 35: "the alleged right of intervention as the manifestation of a policy of force, such as has, in the past, given rise to most serious abuses and such as cannot, whatever be the present defects in international organization, find a place in international law. Intervention is perhaps still less admissible in the particular form it would take here; for, from the nature of things, it would be reserved for the most powerful States, and might easily lead to perverting the administration of international justice itself.»

33 Véase, por ejemplo, mi artículo «La responsabilidad de proteger y el uso de la fuerza en el debate sobre la reforma de la ONU", Papeles de cuestiones internacionales, núm. 91, 2005, pp. 33 ss.

34 Report of the International Commission on Intervention and State Sovereignty, The Responsibility to Protect, 2001, disponible en http://www.iciss.ca/pdf/CommissionReport.pdf. Una síntesis en G. EVANS y M. SAHNOUN, «The Responsibility to Protect», Foreign Affairs, vol. 81, núm. 6, 2002, pp. 99 ss. 
como una norma emergente de derecho internacional por el Grupo de alto nivel sobre las amenazas, los desafíos y el cambio en su propio informe preparado para el Secretario General de $\mathrm{Na}$ ciones Unidas $^{35}$. El antiguo Secretario General Kofi Annan hizo suya la idea en su importante informe «Un concepto más amplio de libertad: desarrollo, seguridad y derechos humanos para todos» ${ }^{36}$, donde afirmó que «no puede aceptarse que, cuando la comunidad internacional se encuentre frente al genocidio o los abusos en masa contra los derechos humanos, las Naciones Unidas se mantengan al margen y dejen que los acontecimientos sigan su curso hasta el final, con consecuencias desastrosas para muchos millares de personas inocentes» ${ }^{37}$. Por ese motivo, en su informe recomienda que «debemos asumir la responsabilidad de proteger y, cuando sea necesario, debemos actuar en consecuencia ${ }^{38}$. Todos estos

35 «Un mundo más seguro: la responsabilidad que compartimos", Documento de las Naciones Unidas A/59/565, de 2 de diciembre de 2004, párrafos 199-203, disponible en http://www.un.org/spanish/ secureworld/index.html. En este informe se habla de la responsabilidad de proteger como una norme emergente, como «la norma que se está imponiendo en el sentido de que existe una responsabilidad internacional colectiva de proteger» (párrafo 203).

36 Documento de las Naciones Unidas A/59/2005, disponible en http://www. un.org/spanish/largerfreedom.

37 Un concepto más amplio de libertad, ibid., párrafo 134.

38 Un concepto más amplio de libertad, ibid., párrafo 135. informes hacen hincapié en la idea de soberanía como responsabilidad y hacen recaer la principal carga en los Estados, que tienen la obligación de proteger a su población. El problema está en los casos en que «las autoridades nacionales no estén dispuestas a proteger a sus ciudadanos o no puedan hacerlo»; en esos casos se trasladaría «a la comunidad internacional la responsabilidad de utilizar medios diplomáticos, humanitarios y de otro tipo para contribuir a proteger los derechos humanos y el bienestar de la población civil» ${ }^{39}$. Por supuesto, el alcance y los límites de la responsabilidad de proteger son controvertidos, pero en cualquier caso es un concepto que ofrece una imagen clara de la transformación del Derecho internacional y los retos a la igualdad soberana y sus consecuencias normativas.

Otro caso reñido radica en el choque entre las inmunidades jurisdiccionales de los Estados y los derechos de las víctimas de violaciones graves de derechos humanos ${ }^{40}$. Un embate que se produce por la contraposición entre

39 Un concepto más amplio de libertad, ibid., párrafo 135. La responsabilidad de proteger a las poblaciones del genocidio, los crímenes de guerra, la depuración étnica y los crímenes de lesa humanidad fue incorporada al Documento Final de la Cumbre Mundial 2005 (resolución de la Asamblea General A/Res/60/1, de 24 de octubre de 2005, párrafos 138-139) y luego fue reafirmada por el propio Consejo de Seguridad en su resolución S/ RES/1674 (2006), sobre protección de civiles en los conflictos armados.

40 Me he ocupado extensamente de este tema 
"dos conjuntos de normas que tienen objetivos muy diferentes» ${ }^{41}$. En efecto, las inmunidades jurisdiccionales de los Estados, que son fundamentalmente un límite al ejercicio de la jurisdicción territorial de los Estados establecido por el derecho internacional, tienen por objeto hacer respetar el principio de igualdad soberana de los Estados; en cambio, los derechos humanos protegen derechos y libertades fundamentales de las personas frente a los Estados y garantizan una reparación efectiva en el caso de que se produjera una violación de esos derechos. Esta pugna entre dos objetivos legítimos del derecho internacional se ha visto reflejada en los últimos años en casos concretos donde individuos demandan civilmente a Estados extranjeros ante tribunales nacionales alegando violaciones graves de derechos humanos, como la tortura o el genocidio, por las que solicitan una reparación efectiva. Un caso paradigmático que ilustra esta tensión es el asunto Al-Adsani, que tras ser decidido a favor de la aplicación de la inmunidad jurisdiccional de Kuwait en los tribunales del Reino Unido, fue confirmado en una senten-

en mi libro Inmunidad del Estado y derechos humanos, Civitas, Navarra, 2007.

41 Comisión de Derecho Internacional, Fragmentación del derecho internacional: dificultades derivadas de la fragmentación y expansión del derecho internacional, U.N. Doc. A/CN.4/L/.702, 18 de julio de 2006; y U.N. Doc. A/CN.4/L/.682, 13 de abril de 2006 (completado por Martti KOSKENNIEMI), párrafo 24, disponible en http://untreaty.un.org/ilc/texts/1_9.htm. cia del Tribunal Europeo de Derechos Humanos, aunque por una exigua minoría ${ }^{42}$. No es este el lugar para entrar en un análisis de las excepciones a las inmunidades jurisdiccionales de los Estados en los tribunales de otros Estados y, especialmente, de si cabría una excepción basada en violaciones graves de derechos humanos ${ }^{43}$. Mi intención, como advertí en la introducción, es ilustrar cómo los derechos de los Estados basados en su igualdad soberana se ven socavados por la evolución del derecho internacional, que en este caso se refleja en la reconsideración de la inmunidad de jurisdicción, que está sometida a un importante reto para mantener su vigencia como regla de Derecho internacional aplicable a los Estados demandados en tribunales de otros Estados cuando los demandados han sido autores de violaciones graves de derechos humanos, especialmente

42 Sentencia del Tribunal Europeo de Derechos Humanos en el caso Al-Adsani v. United Kingdom, de 21 de noviembre de 2001, asunto núm. 35763/97, decidido por el voto de 9 jueces contra 8 . Este caso ha generado una discusión enorme; una relación de comentarios y críticas puede consultarse en mi trabajo «Sobre la emergencia de una excepción a las inmunidades jurisdiccionales de los Estados ante las violaciones graves de derechos humanos", Cursos de Derecho Internacional y Relaciones Internacionales de Vitoria-Gasteiz 2007, Bilbao, 2008, p. 208, nota 5; y un análisis más detallado en Inmunidad del Estado y derechos humanos, supra nota 40, capítulo IV.

43 Nuevamente remito a mi libro Inmunidad del Estado y derechos humanos, supra nota 40. 
cuando las violaciones se refieran a normas de derechos humanos con carácter de ius cogens, en particular la prohibición de la tortura ${ }^{44}$.

A estas alturas ya es manifiesto que la igualdad no equivale a identidad ${ }^{45}$. Con los ejemplos anteriores he intentado mostrar, además, que las transformaciones del derecho internacional hacen que ni siquiera los derechos inherentes a la soberanía formal del Estado salgan intactas de los desafíos y retos que dichos cambios suponen. Es cierto que, en algunos casos, los pretendidos cambios no son tales o no se justifican en relación con la sociedad internacional plural en la que el derecho internacional debe operar. Un ejemplo claro de este tipo viene de la mano de las propuestas que sostienen la virtualidad de un principio democrático para el derecho internacional, que socavaría claramente la igualdad jurídica de los Estados ${ }^{46}$. En otros ca-

44 Sobre la tortura, en especial, A. CLAPHAM, «The jus cogens Prohibition of Torture and the Importance of Sovereign State Immunity", en M.G. KOHEN (ed.), Promoting Justice, Human Rights and Conflict Resolution through International Law: liber amicorum Lucius Caflisch, La Haya, Nijhoff, 2007; K. PARLETT, «Immunity in Civil Proceedings for Torture: The Emerging Exception", European Human Rights Law Review, 2006, pp. 49 ss.

45 A. REMIRO BROTÓNS, R. RIQUELME CORTADO, J. DÍEZ-HOCHLEITNER, L. PÉREZ PÉREZ-PRAT DURBÁN Y E. ORIHUELA CALATAYUD, Derecho internacional, Tirant lo Blanc, Valencia, 2007, p. 146.

46 A. REMIRO BROTÓNS, «Soberanía del Estado, libre determinación de los pueblos sos, las excepciones a la igualdad provienen del propio consentimiento de los Estados, como cuando los Estados prestan su consentimiento en obligarse por tratados que contienen normas que otorgan derechos y prevén deberes distintos para diversos tipos de Estados $^{47}$ o cuando los Estados se adhieren a una organización internacional ${ }^{48}$ que opta por soluciones basadas en la igualdad material de los Estados frente a la igualdad formal. Esto ocurre con el sistema de votación de las instituciones financieras y monetarias internacionales, el Banco Mundial ${ }^{49}$ y del $\mathrm{FMI}^{50}$, así como con la famosa

y principio democrático", en F. MARIÑO MENÉNDEZ (ed.), El Derecho internacional en los albores del siglo XXI: Homenaje al Profesor Juan Manuel CastroRial Canosa, Trotta, Madrid, 2002, pp. 545 ss.

47 Véase infra apartados III.B y III.C, donde me ocupo de los tratados desiguales y de las responsabilidad diferenciada, respectivamente.

48 Cf.A.D. EFRAIM, Sovereign (In)Equality in International Organizations, Nijhoff, La Haya, 2000 (que aboga por no aplicar el principio ortodoxo de igualdad soberana en Derecho internacional institucional).

49 Se afirma que su sistema de gobierno está anclado en el pasado, porque han cambiado los papeles de los Estados deudores y capitalistas, y se han incorporado fuentes privadas y Estados emergentes como actores relevantes del sistema. Las ONG y los Estados en desarrollo proponen un cambio hacia un sistema de paridad entre países avanzados y en desarrollo, pero en realidad no se atisba un cambio en este sentido.

50 Otro sistema controvertido en el que los países del G-8 tienen la mayoría de las acciones con derecho a voto, y por tanto, 
desigualdad intrínseca del derecho de veto de los cinco miembros permanentes del Consejo de Seguridad de las Naciones Unidas. Conviene precisar algo más estas ideas respecto del Consejo de Seguridad, por su importancia y su notoriedad.

Efectivamente, el derecho de veto en el Consejo de Seguridad es percibido como la desigualdad más palpable del sistema jurídico internacional. Sin embargo, desde el punto de vista del derecho internacional no representa tanto un problema que afecte al principio de igualdad soberana de los Estados, sino más bien un problema de legitimidad, eficacia y transparencia en el sistema de seguridad colectiva. Esto es así porque los privilegios que suponen el derecho de veto en el Consejo de Seguridad de los cinco miembros permanentes ${ }^{51}$ se basan en el consentimiento de los Estados en un tratado internacional constitutivo de una organización internacional como es la Carta de las Naciones Unidas, por lo tanto se trata de una limitación consentida y voluntaria de la igualdad soberana de los Estados.

Los esfuerzos por mejorar la eficacia y representatividad del Consejo de Seguridad han sido tan constantes

están en posibilidades de determinar las políticas del FMI. Además, cualquier política del FMI necesita un $85 \%$ de los votos para ser aprobada, lo que le confiere a Estados Unidos un veto efectivo.

51 China, Estados Unidos de América, Francia, Reino Unido y Rusia. y numerosos como fútiles ${ }^{52}$. Estos empeños se concentran especialmente en la composición ${ }^{53}$ y las formas de tomar decisiones $^{54}$ en el Consejo de Seguri-

52 Para un estudio de dichas propuestas: $\mathrm{R}$. RIQUELME CORTADO, La reforma del Consejo de Seguridad de la ONU, Dykinson, Madrid, 2000; y los trabajos de B. FASSBENDER, UN Security Council Reform and the Right of Veto: A Constitucional Perspective, Kluwer, The Hague, 1998, y «Pressure for Security Council Reform», en D.M. MALONE (ed.), The UN Security Council, Lynne Reinner, Boulder and London, 2004, pp. 341 ss.

53 En relación con la composición del Consejo se propugna una revalorización de la letra del artículo 23 de la Carta, que se refiere al criterio distribución geográfica equitativa, pero también se menciona el criterio de la contribución al mantenimiento de la paz y la seguridad internacionales por parte de los Estados para la elección de los miembros no permanentes. Esa contribución se debería evaluar teniendo en cuenta aspectos financieros, militares y diplomáticos, como «las cuotas para el presupuesto prorrateado de las Naciones Unidas, la participación en operaciones de la paz, las aportaciones a actividades voluntarias de las Naciones Unidas en apoyo de los objetivos y mandatos de las Naciones Unidas» e incluso el porcentaje de ayuda oficial al desarrollo. Un mundo más seguro, supra nota 35, párrafo 249. Aunque resultaría bastante difícil que una revisión de ese tipo pudiera aceptarse, ya que estos criterios de selección podrían afectar negativamente a los actuales -y posibles futurosmiembros permanentes del Consejo de Seguridad. Por ejemplo: ¿cómo se estimaría el hecho de que los miembros del Consejo de Seguridad controlen el mercado mundial de armamentos?

54 Véase S. D. BAILEY y S. DAWS, The Procedure of the Security Council, Clarendon, Oxford, 3rd ed. 1998. 
dad. Esas fórmulas parten de la necesidad de una mayor representatividad en el Consejo de Seguridad, especialmente de países menos desarrollados, pero tratan a la vez de no afectar a su eficacia y hacer de él un órgano más democrático, que rinda cuentas de su actuación. Por ejemplo, en el informe Un mundo más seguro hay fórmulas que proponen una ampliación distribuida equitativamente entre África, Asia y el Pacífico, Europa y América, aumentando en nueve los puestos del Consejo y convirtiéndolo, por tanto, en un órgano de veinticuatro miembros. El problema es cuántos de esos miembros nuevos serían permanentes. Ahí las propuestas difieren: un modelo propone crear seis nuevos puestos permanentes ${ }^{55} \mathrm{y}$ tres no permanentes de dos años de duración, mientras que el otro modelo no propone la creación de ningún otro puesto permanente, sino de ocho nuevos miembros de una categoría nueva, con un mandato de cuatro años renovables, y un nuevo miembro no permanente con un mandato como el actual de dos años no renovables.

En relación con el derecho de veto de los cinco miembros permanentes

55 Previsiblemente, aunque con muchos obstáculos, se habla de Japón, Alemania, Brasil, India, Sudáfrica y Nigeria. Para un breve ensayo sobre los miembros permanentes y los miembros electos en el actual Consejo de Seguridad, se puede consultar el artículo de K. MAHBUBANI, "The Permanent and Elected Members", que aparece en el libro de David MALONE, supra nota 52, pp. 253 ss. del Consejo, cuyo uso o amenaza de uso sigue siendo relevante para el sistema de seguridad colectiva ${ }^{56}$, las propuestas son muy tímidas. Aunque el veto es calificado de anacrónico y antidemocrático, los expertos recomiendan no tocar el poder de veto de los que ya lo tienen ni ampliar el veto en cualquiera de las opciones de reforma del Consejo de Seguridad ${ }^{57}$. Esta es, sin duda, una posición realista, basada en argumentos razonables tanto desde la perspectiva del Consejo de Seguridad como de los Estados que lo componen. Desde el punto de vista del Consejo, se ha hablado del veto como de una "válvula de escape», que permite que no se tomen decisiones que posteriormente no podrán cumplirse por falta del apoyo o los medios necesarios para dicho fin. Si se mira desde el punto de vista de los Estados, no cabe esperar que los miembros permanentes acepten una reforma en la que vean menoscabado o pierdan el poder de veto ${ }^{58}$. De hecho, el veto fue una forma de involucrar a los poderosos en una organización que quizás de otra forma no hubieran

$56 \mathrm{Al}$ respecto, puede consultarse, por ejemplo, P. WALLENSTEIN y P. JOHANSSON, «Security Council Decisions in Perspective», en D.M. MALONE (ed.), supra nota 52, pp. 17 ss.

57 Un mundo más seguro, supra nota 38, párrafo 256.

58 Hay que tener en cuenta que cualquier reforma de la Carta de las Naciones Unidas necesita el voto afirmativo de los miembros permanentes del Consejo de Seguridad.Artículo 108 de la Carta de las Naciones Unidas. 
aprobado $^{59}$. En cambio, se proponen fórmulas que mejoren la transparencia y eficacia de la toma de decisiones sin tratar de reformar la Carta para modificar el derecho de veto. Por ejemplo, se ha sugerido que se instituya un sistema de «voto indicativo» respecto de una propuesta de decisión ${ }^{60}$, algo que permitiría evitar posteriores vetos, en la medida en que constituiría una especie de votación oficiosa que luego debería ser adoptada oficialmente para que constituyese una verdadera decisión. También se ha sugerido hacer recaer la carga de la prueba para justificar un veto en los miembros permanentes que decidan utilizarlo, o exigir dos vetos concurrentes para bloquear una decisión, o reducir las circunstancias en que los vetos pudieran tener valor, o en general tratar de reducir el «déficit deliberativo ${ }^{61} \mathrm{del}$ Consejo. Son todas propuestas pragmáticas, que tratan

59 Por ejemplo, Cordell HULL, antiguo Secretario de Estado de EE.UU., declaró que "su Gobierno no permanecería un día en las Naciones Unidas sin el poder de veto» (citado por MAHBUBANI, supra nota 55, p. 255).

60 Un mundo más seguro, supra nota 35, párrafo 257.

61 I. JOHNSTONE, «Legislation and Adjudication in the UN Security Council: Bringing Down the Deliberative Deficit», American Journal of International Law, vol. 102, 2008, pp. 275 ss. Este autor propone medidas que lleven a generar consultas inclusivas en el Consejo, justificación pública y revisión independiente de las decisión del Consejo (p. 303 ss.), con el objeto de cultivar una práctica que suponga la utilización de un discurso razonado e imparcial. de restringir el veto mediante cambios procedimentales que no supongan una reforma de la Carta de Naciones Unidas.

\section{B. La igualdad soberana de los Estados en la creación del Derecho internacional}

La reflexión que debe primar en materia de fuentes de producción del derecho internacional en relación con el principio de igualdad soberana concierne al valor del consentimiento de los Estados en la creación de obligaciones internacionales. El consentimiento de los Estados ha sido siempre considerado como la piedra angular del derecho internacional. Se trata de una expresión de la igualdad formal, no material, de los Estados que en el derecho de los tratados se traduce esencialmente en la capacidad de consentir en obligarse por un tratado y en la regla que establece que «un tratado no crea obligaciones ni derechos para un tercer Estado sin su consentimiento» ${ }^{62}$.

Son elementos derivados de los contenidos de la igualdad soberana de los Estados en un sentido formal. En realidad, una concepción distinta del derecho internacional podría haber permitido la incorporación de

62 Artículo 34 de la Convención de Viena de Derecho de los Tratados de 1969. Sobre el Derecho de los tratados véase, por todos, el completo estudio de A. REMIRO BROTÓNS, Derecho de los tratados, Tecnos, Madrid, 1987. 
elementos de igualdad material en los tratados, pero no ha ocurrido así. Un ejemplo muy gráfico es el de los tratados desiguales. A pesar de la insistencia de China para conseguir la nulidad o terminación de los tratados que se produjeron como consecuencia de las Guerras del Opio ${ }^{63}$ de 1839-1842 y 1856-1860, no se ha aceptado en derecho internacional el concepto de tratados desiguales ${ }^{64}$ como causa de nulidad o terminación. Habrá que recurrir a las causales de coacción en el representante del Estado ${ }^{65}$ para la nulidad o al cambio fundamental en las circunstancias $^{66}$ como causa de terminación

63 Mediante estas guerras los británicos forzaron la apertura de China al comercio británico con el objetivo específico de exportar opio. Esta droga era fundamental para el comercio británico colonial; se exportaba desde la India en importantes cantidades (una tonelada por día hasta 1839), y permitía pagar las importaciones de té y seda. La Guerras produjeron los tratados de Nankin de 1842, Tianjin 1858 y Pekín 1860. Estos tratados preveían la apertura de ciertos puertos, conocidos como «treaty ports», para la importación de opio. El profesor LOWE ha dicho con certeza que ha pasado generalmente desapercibida la ironía de dar en 2002 al Reino Unido la responsabilidad de liderar la campaña contra las drogas para la erradicación del opio en Afganistán. V. LOWE, International Law, Oxford University Press, Oxford, 2007, p. 189.

64 Sobre los tratados desiguales véase la entrada de W. MORVAY en la Encyclopedia of Public International Law, supra nota 15.

65 Artículo 51 de la Convención de Viena de Derecho de los Tratados de 1969.

66 Artículo 62 de la Convención de Viena de Derecho de los Tratados de 1969. de un tratado. Pero la desigualdad en sí misma se trata como un problema de legitimidad más que de legalidad ${ }^{67}$.

Sin embargo, el requisito del consentimiento se modula y hasta queda en un plano subordinado como consecuencia de la aparición en derecho internacional de obligaciones que no pueden desconocerse por la mera falta de consentimiento de uno o más miembros de la comunidad internacional, como las obligaciones de ius cogens ${ }^{68}$. Este tipo de obligaciones no pueden dejarse de lado como consecuencia del consentimiento. Así, el artículo 53 de la Convención de Viena sobre el derecho de los Tratados establece que:

«Es nulo todo tratado que, en el momento de su celebración, esté en oposición con una norma imperativa de derecho internacional general. Para los efectos de la presente Convención, una norma imperativa de derecho internacional general es una norma aceptada y reconocida por la comunidad internacional de Estados en su conjunto como norma que no admite

67 Hoy, por ejemplo, algunos autores plantean la cuestión relativa a la legitimidad del desequilibrio entre obligaciones y derechos de países desarrollados y en desarrollo dentro del Acuerdo sobre los aspectos de los Derechos de Propiedad Intelectual relacionados con el Comercio (ADPIC) dentro del sistema de la OMC. Para una lectura de los artículos del ADPIC en clave de desarrollo, véase Resource Book on TRIPS and Development, Cambridge, Cambridge University Press, 2005.

68 Véase, por ejemplo, A. ORAKHELASHVILI, Peremptory Norms in International Law, Oxford University Press, Oxford, 2006. 
acuerdo en contrario y que sólo puede ser modificada por una norma ulterior de derecho internacional general que tenga el mismo carácter».

Con independencia de los problemas de identificación de las normas de ius cogens, su aceptación como normas de Derecho internacional de jerarquía superior que no admiten acuerdo en contrario es un dato concluyente de la inoperatividad del poder irrestricto del consentimiento basado en el principio de igualdad soberana respecto de la obligatoriedad de este tipo de normas.

Pero no sólo las normas de ius cogens pertenecen a esta categoría de obligaciones de los Estados que demuestran que el consentimiento no es la base exclusiva de la creación de normas obligatorias en derecho internacional. Se puede hablar también de obligaciones surgidas sin el consentimiento del Estado o incluso en contra de su voluntad ${ }^{69}$. En efecto, hoy contamos con normas obligatorias emanadas de organismos internacionales, donde el consentimiento no siempre se expresa específicamente respecto de cada disposición obligatoria para los Estados, como ocurre con las decisiones, las directivas y los reglamentos de las Comunidades Europeas. Un caso muy llamativo de estas características son las resoluciones obligatorias del Consejo de Seguridad con carácter institucional o legislativo. Entre las

69 C. TOMUSCHAT, supra nota 26. primeras se encuentran las resoluciones por las que se crean los tribunales penales internacionales de carácter especial para la Antigua Yugoslavia ${ }^{70}$ o para Rwanda ${ }^{71}$; entre las segundas están, por ejemplo, las decisiones sobre lucha contra el terrorismo internacional adoptadas tras los atentados contra las Torres Gemelas en Nueva York el 11 de septiembre de 200172, que establecen un régimen de obligaciones específicas para todos los Estados en esta materia, incluyendo algunas obligaciones que nunca pudieron acordarse entre los Estados en foros de negociación internacional como la Asamblea General de las Naciones Unidas.

Estas prácticas revelan los retos a los que se encuentra sometido el principio en los órganos de las organizaciones internacionales. En efecto, más allá de la cuestión ya señalada respecto de la validez de las limitaciones de derechos soberanos por parte de los Estados al crear o adherirse a una organización internacional, en su sentido más acabado el principio de igualdad soberana requeriría la utilización de la regla «un Estado un voto». Esa regla está prevista, por ejemplo, en el procedimiento de adopción de decisiones

70 Resolución del Consejo de Seguridad S/ RES/827 (1993), de 25 de mayo de 1993.

71 Resolución del Consejo de Seguridad S/ RES/955 (1994), de 8 de noviembre de 1994.

72 Especialmente, la resolución S/RES/1773 (2001), adoptada sólo unos días después de los atentados terroristas en Estados Unidos de América. 
de la Asamblea General de las Naciones Unidas y es muy importante en la medida en que mediante sus votos los Estados expresan su aceptación de prácticas que podrían conformar normas de derecho internacional consuetudinario. Pero aun en la Asamblea General se observan desviaciones en la práctica respecto del principio «un Estado un voto» y existen presiones de reforma basadas en la falta de representatividad democrática que encarna el principio. Las desviaciones fueron inevitables como consecuencia de la irrelevancia de las mayorías en ciertas votaciones de la Asamblea, que adquieren especial importancia en la adopción de decisiones en materia de igualdad distributiva ${ }^{73}$. Esta constatación ha hecho necesario recurrir al consenso como un método alternativo de decisión frente a la regla «un Estado un voto". Sin embargo, aunque las apariencias pudieran resultar engañosas, el consenso es un método muy bien avenido con la igualdad soberana en un sentido formal $y$, particularmente, con el consentimiento, en la medida que el consenso establece un método de toma de decisiones basado en una regla «súper-mayoritaria», que exige la ausencia de votos negativos. Es precisamente por esa razón por la que el consenso también se ha introducido como una práctica en el Consejo de Seguridad mediante las declaraciones

73 Véase supra nota 29 sobre el Derecho internacional del desarrollo. presidenciales $^{74}$. Si bien esta práctica parece respetuosa del principio de igualdad soberana de los Estados en un sentido formal, cabe mostrar cierto escepticismo sobre en qué medida los Estados débiles pueden hacer valer su voz y criterios en este tipo de procedimientos ${ }^{75}$.

Las presiones de reforma de la regla «un Estado un voto» se basan en argumentos de representación democrática, eficacia y equidad. Este tipo de argumentos se aplican a la Asamblea General de las Naciones Unidas, pero también a otras instituciones internacionales como la Organización Mundial del Comercio ${ }^{76}$. Esta preocupación está conectada a un problema de inclusión. En efecto, como ha puesto de manifiesto el profesor Thomas Frank, el discurso del Derecho internacional tiene dos características dominantes que desafían a la equidad: primero, el discurso tiende a dar a cada Estado una voz igual; segundo, la tendencia es que esa voz se otorgue sólo a los representantes de los gobiernos ${ }^{77}$. Esta

74 S. C. HULTON, "Council Working Methods and Procedure», en D.M. MALONE (ed.), supra nota 52, pp. 237 ss., esp. 237339. Las declaraciones presidenciales se hacen en público por parte del Presidente de turno del Consejo y necesitan el consenso de los miembros del Consejo respecto de todas y cada una de sus palabras.

75 Véase Human Development Report 2002, Oxford University Press, Nueva York y Oxford, 2002, pp. 127 ss.

76 J. JACKSON, Soberanía, OMC y los fundamentos cambiantes del Derecho internacional, Marcial Pons, Madrid, 2009.

77 T.M. FRANCK, Fairness in International 
última aseveración, muy apropiada y generalmente veraz, revela un problema de inclusión como un proceso que afecta a la subjetividad soberana en el derecho internacional ${ }^{78}$, que está ligado a la legitimidad de algunas normas internacionales. En efecto, la globali-

Law and Institutions, Oxford University Press, Oxford, 1995, pp. 478-481. Como una forma de equilibrar la balanza de participación con el fin de producir un resultado más justo, Franck sugiere que se cree un foro de representación directa de los pueblos, por ejemplo, mediante la creación de dos cámaras en la Asamblea General de las Naciones Unidas, una de los pueblos y otra de los gobiernos. La cámara de los pueblos se elegiría directamente sobre la base de un sistema proporcional en relación con la población de cada Estado. Las decisiones importantes se tomarían por simple mayoría de ambas cámaras. Es interesante destacar que, dado que la Asamblea General no tiene poder de adoptar decisiones obligtorias, en realidad se trataría de un cambio que afectaría a la forma y la estructura del Derecho internacional de una manera delicada, a través de un cambio en el discurso internacional debido a una mayor inclusión de participantes. El problema de este tipo de propuestas de democracia mundial o cosmopolita radica especialmente en la forma de hacerlas operativas. Cf., por ejemplo, L. RODRÍGUEZ ABASCAL, "El debate sobre los derechos de grupo", en E. DÍAZ y J.L. COLOMER (eds.), Estado, justicia, derechos, Alianza, Madrid, 2002, pp. 409 ss., donde el autor considera objeciones conceptuales, normativas y pragmáticas al reconocimiento y puestas en práctica de los derechos de grupo.

78 J. NIJMAN, «Sovereignty and Personality: A Process of Inclusion», en G. KREIJEN (ed.), State, Sovereignty and International Governance, Oxford University Press, Oxford, 2004, pp. 109 ss. zación ha traído consigo el crecimiento en número y relevancia de actores no estatales ${ }^{79}$ que participan en procesos que desbordan al Estado, escapan a sus modos de legitimación y se posan al margen de los medios de aplicación del derecho articulados por el derecho internacional interestatal, poniendo en evidencia su insuficiencia. Esos actores no estatales tienen difícil cabida en los procesos de creación de tratados y costumbres internacionales, por lo que dichos procesos padecen un déficit de participación que se manifiesta en el hecho de que vivimos en un mundo de múltiples actores donde la producción normativa de Derecho internacional ocurre fundamentalmente en un nivel intergubernamental. Esta situación, sin embargo, está cambiando en algunos ámbitos jurídicos de la mano de procesos de creación normativa que se generan, tienen éxito o son derrotados como consecuencia de la acción coordinada de la sociedad civil a través de organizaciones no gubernamentales ${ }^{80}$.

\section{La igualdad soberana de los Estados en la aplicación del derecho internacional}

Tal y como avancé en la introducción, al igual que con los sujetos y la creación del derecho internacional, la

79 L. PÉREZ-PRAT DURBÁN, Sociedad civil $y$ Derecho internacional, Tirant lo Blanc, Valencia, 2004.

80 Ibid., especialmente, capítulo 6, sobre la acción normativa innovadora de los actores no estatales, pp. 265 ss. 
referencia a la aplicación del derecho internacional es simplemente ilustrativa para mostrar el tipo de retos a los que se enfrenta el principio de igualdad soberana de los Estados en contextos interdependientes y globalizados. Tres tipos de casos normativos bastarán para mostrar estos retos.

El primero se refiere a regímenes de responsabilidad común pero diferenciada. En efecto, en ocasiones los Estados soberanos se obligan por tratados que prevén regímenes de responsabilidad diferenciada, con derechos y deberes desiguales para las partes. Es lógico que así sea en tanto resulta racional que la desigualdad real se vea reflejada en el reparto de responsabilidades con el fin de asegurar la eficacia en el logro de objetivos concretos a través de la cooperación y de acuerdo con las capacidades de cada Estado en ámbitos como el derecho económico internacional ${ }^{81} \mathrm{o}$ el derecho internacional del medio ambiente ${ }^{82}$. Los regí-

81 En la OMC, por ejemplo, existen diversos mecanismos de equidad, como la Parte IV del GATT, los Sistemas de Preferencias Generalizadas o la cláusula de habilitación adoptada el 28 de noviembre de 1979 (Enabling Clause). Véase M. MATSUSHITA, T. SCHOENBAUM y P. MAVROIDIS, The World Trade Organization, Oxford University Press, Oxford, $2^{\text {nd }}$ ed. 2006, pp. 763 ss.; C.MICHALOPOULOS, Developing Countries in the WTO, Palgrave, Londres, 2001.

82 L. RAJAMANI, Differential Treatment in International Environmental Law, Oxford University Press, Oxford, 2006.

Véase también el curso de Á.J. RODRIGO HERNÁNDEZ, «Nuevas técnicas menes de responsabilidad diferenciada no siempre se vuelcan a favor de los menos desarrollados, como ocurre con ciertas organizaciones internacionales de seguridad colectiva ${ }^{83}$ o con los regímenes convencionales de control de armamentos y desarme ${ }^{84}$, que les otorgan un mayor poder y una responsabilidad más grave a los Estados más poderosos e industrializados.

Los regímenes que favorecen a los menos desarrollados utilizan mecanismos de diversa índole, que pueden comprender transferencia tecnológica, exenciones, ayudas financieras o periodos de transición con mayores plazos para la efectiva implementación de las obligaciones previstas en los tratados. Esos instrumentos de tratamiento diferenciado tienen por finalidad ayudar a los Estados más desfavorecidos a cumplir con las obligaciones establecidas, pero también se basan en el reconocimiento de que algunos Estados son más responsables que otros en la creación de ciertos problemas comunes, como la contaminación ambiental, y que por ese motivo deben asumir

jurídicas para la aplicación de los tratados internacionales de medio ambiente», en Cursos de Derecho Internacional y Relaciones Internacionales de Vitoria-Gasteiz 2001, pp. 155 ss.

83 Como ocurre con el Consejo de Seguridad de las Naciones Unidas. Véase supra apartado III.A.

84 Por ejemplo, el Tratado sobre la No proliferación de armas nucleares de 1968, que establece dos categorías de Estados Partes, los Estados que poseen armas nucleares y los demás. 
más cargas. Además, el tratamiento diferenciado es también un sistema de incentivos para el cumplimiento de obligaciones internacionales cuya observancia es necesaria para preservar bienes públicos globales e intereses comunes de los Estados.

El segundo caso se refiere a una situación producida por la arquitectura operativa del derecho internacional. Ciertamente, como consecuencia de la descentralización y el desdoblamiento funcional del derecho internacional, en el ámbito de la aplicación del derecho es dónde las diferencias de poder sustantivo tiene más notoriedad y pueden crear diferencias importantes tanto en el modo de cumplir con las obligaciones internacionales, como en la capacidad de violar ciertas normas que sólo los poderosos están en condiciones de no respetar, como la prohibición de las intervenciones armadas, o de utilizar los mecanismos del derecho internacional con toda su eficacia, como ocurre con las contramedidas de carácter económico o comercial ${ }^{85}$. Esta última situación es particularmente sugerente y por ello la solución de diferencias en el sistema de la OMC adquiere su mayor virtualidad en las etapas posteriores a la adopción del informe de los Grupos Especiales o del Órgano de Apelaciones cuando los Estados involucrados en la diferencia tienen una capacidad similar de adoptar

85 Véase mi «Introducción al sistema de solución de diferencias de la OMC», Revista Jurídica de la UAM, núm. 5, 2001, pp. 253 ss., p. 266. medidas compensatorias y represalias comerciales ante los incumplimientos de los Estados perdedores ${ }^{86}$.

El tercer caso enfrenta al principio de igualdad soberana de los Estados al proceso de individualización del derecho internacional, como sucede con el derecho internacional de las inversiones $^{87}$. En este ámbito del derecho internacional se ha producido una sustitución de los Estados, como protectores de los intereses nacionales en el ex-

86 En el artículo 22.3.b) y c), el Entendimiento sobre Solución de Diferencias de la OMC ha previsto las compensaciones y la suspensión de concesiones u otras obligaciones de forma cruzada, es decir, dirigidas a sectores o incluso Acuerdos diferentes a los que originan la reclamación como una vía para conseguir mayor eficacia en las contramedidas tendentes a cesar la situación de inconsistencia con las normas de los Acuerdos OMC (por ejemplo, un Estado sufre una infracción, anulación o menoscabo de sus derechos en materia agrícola $y$, tras comprobar que en ese sector no tendrá efecto su medida de compensación, decide dirigirla a los derechos de propiedad intelectual protegidos en el ADPIC). Sin embargo, las diferencias de poder son determinantes aún en el caso de las represalias cruzadas y podría decirse que sólo los Estados poderosos estarían en condiciones de utilizarlas de una manera efectiva; es más, podría incluso decirse que es una herramienta adicional de los Estados poderosos.

87 Sobre esta dinámica área del Derecho internacional, véase, por ejemplo, R. DOLZER y C. SCHREUER, Principles of International Investment Law, Oxford University Press, Oxford, 2008; M. SORNARAJAH, The International Law on Foreign Investment, Cambridge University Press, Cambridge, 2da ed 2004. 
tranjero $^{88}$, por los individuos y empresas, que se encuentran habilitados para defender sus propios intereses frente a los Estados de forma directa y ante órganos de carácter privado que han reemplazado a los poderes públicos en la solución de las controversias internacionales en materia de inversión ${ }^{89}$. La legitimación de los inversores para evitar los recursos judiciales internos y acceder directamente a métodos de solución de controversias internacionales tiene lugar especialmente mediante una red de más de dos mil tratados bilaterales de protección de las inversiones en el extranjero, que se caracterizan por prever el recurso por parte de los particulares a arbitrajes internacionales con el fin de resolver las controversias entre los Estados receptores de la inversión y los inversores extranje$\operatorname{ros}^{90}$. Esta práctica, que se separa de la solución tradicional de controversias entre Estados, plantea problemas graves de legitimidad desde las perspectivas de la estructura institucional ${ }^{91} \mathrm{de}$

88 J. Díez-Hochleitner, «Protección diplomática v. arbitraje de inversiones», en El Derecho internacional: normas, hechos y valores. Liber amicorum J.A. Pastor $R i$ druejo, Universidad Complutense de Madrid, Madrid, 2005, pp. 469 ss.

89 W. MATTLI, 'Private Justice in a Global Economy: From Litigation to Arbitration', International Organization, vol. 55, 2001, pp. 919 ss.

90 C. MCLACHLAN, L. SHORE, M. WEINIGER, International Investment Arbitration, Oxford University Press, Oxford, 2007.

91 G. VAN HARTEN, Investment Treaty Arbitration and Public Law, Oxford Uni- los procesos arbitrales de inversión y de la jurisprudencia ${ }^{92}$ de los tribunales arbitrales de inversión. Efectivamente, los tratados bilaterales de inversión delegan funciones judiciales esenciales de derecho público en árbitros privados, que están llamados a decidir importantes cuestiones que surgen de la autoridad soberana de los Estados en las que está en juego el principio de democracia y que suelen afectar a poblaciones enteras de los Estados demandados ${ }^{93}$. Este tipo de procedimiento presupone que los tribunales internos no están capacitados para resolver este tipo de controversias y que, aun cuando lo estuviesen, las resolverían sobre la base de normas y prácticas favorables a los Estados demandados e impactan con el elemento interno de la soberanía de los Estados, eludiendo el principio general de agotamiento de los recursos internos de los Estados antes de pasar a una fase internacio-

versity Press, Oxford, 2007. La percepción de secretismo de estos procesos arbitrales, que resultan contrarios a la necesidad de publicidad de la actividad judicial, ha llegado incluso a las páginas de las revistas de difusión general, como prueba el artículo de The Economist, "Behind Closed Doors» sobre inversión, arbitraje y secreto, de 23 de abril de 2009.

92 S.D. FRANCK, «The Legitimacy Crisis in Investment Treaty Arbitration: Privatizing Public International Law through Inconsistent Decisions", Fordham Law Review, vol. 73, 2004-2005, pp. 1521 ss.

93 Véase, por ejemplo, la serie de casos contra Argentina en el contexto del Centro Internacional de Arreglo de Diferencias relativas a Inversiones en su página http://icsid. worldbank.org/ICSID. 
nal de resolución de una controversia. Esta realidad, esta práctica es compatible con el principio de igualdad soberana de los Estados en un sentido formal, porque los Estados han prestado su consentimiento sobre la base de tratados de promoción y protección de inversiones con el objeto de atraer dichas inversiones y, a cambio, permitirles obtener unas seguridades de protección jurídica internacionales entre las que se encuentra el derecho a llevar una controversia directamente a un arbitraje internacional. La crítica, en consecuencia, debería encaminarse a mejorar el nivel de transparencia de los procedimientos cuando éstos afecten seriamente a la administración pública de los Estados. Junto a la transparencia, también se precisan cambios que supongan asegurar una mayor coherencia de las decisiones y la verdadera independencia de los tribunales, por ejemplo, mediante la creación de un tribunal de apelaciones ${ }^{94} \mathrm{o}$ incluso un tribunal internacional de inversiones ${ }^{95}$.

94 Propuesto por varios autores, incluyendo el ya citado trabajo de Susan FRANCK, supra nota 92. En sentido similar, C.N. BROWER et al., "The Coming Crisis in the Global Adjudicative System», Arbitration International, vol. 19, 2003, pp. 415 ss.

G. VAN HARTEN, «A Case for an International Investment Court», Society of International Economic Law (SIEL) Inaugural Conference 2008, 30 de junio de 2008, disponible en http://ssrn.com/abstract $=1153424$.

\section{Conclusión}

La noción de 'igualdad soberana' es confusa ${ }^{96}$. Es un ideal normativo más que un reflejo de la práctica, que está cada vez más alejado de la realidad por el aumento de participantes sustancialmente diferentes entre sí y el incremento de la interdependencia que complica la posibilidad de tomar decisiones importantes excluyendo su dimensión internacional ${ }^{97}$. En consecuencia, el principio de igualdad soberana de los Estados sobrevive con mucha dificultad en un mundo interdependiente y globalizado compuesto por Estados objetivamente desiguales. Junto a estas dificultades prácticas, la ortodoxia de la igualdad soberana siempre ha tenido detractores. A los autores que hacen lecturas históricas del derecho internacional para demostrar que siempre han existido y existirán jerarquías jurídicas entre Estados ${ }^{98}$ se unen hoy otros autores que intentan, una vez más, reformular los principios fundamentales del derecho internacional sobre la base de características sustantivas de los Estados ${ }^{99}$.

96 H. KELSEN, The Law of the United $\mathrm{Na}$ tions, Stevens, Londres, 1950, pp. 52-53.

97 J. KOKOTT, supra nota 3, párrafo 8.

98 G. SIMPSON, Great Powers and Outlaw States: Unequal Sovereigns in the International Legal Order, Cambridge University Press, Cambridge, 2004.

99 A-M. SLAUGHTER, «International law in a world of liberal states", European Journal of International Law, vol. 6, 1995, pp. 503 ss. 
Ante ese tipo de embates teóricos y de la igualdad soberana de los Estaprácticos provenientes de las lógicas dos sigue siendo una ficción jurídica de globalización, democratización y útil ${ }^{101}$, que conviene mantener vigente privatización en la sociedad interna- en la medida en que la incorporación cional, cuyos efectos sobre el derecho de la desigualdad de los Estados como internacional he intentado mostrar con las ilustraciones precedentes, el principio de igualdad soberana sigue siendo relevante como un puente que conecta a los Estados desiguales ${ }^{100}$. Si bien esta idea puede parecer algo romántica y conservadora respecto al tratamiento de la desigualdad, tengo la convicción de que el principio

100 B. BOUTROS-GHALI, «Le príncipe d'égalité des Etats et les organizations internationales", Recueil des Cours de la Académie de Droit International, 1961-II, p. 69. factor normativo de carácter fundamental no venga apoyada por desarrollos institucionales en la sociedad internacional, que supongan garantías de legalidad y justicia para los Estados más débiles.
101 B. KINGSBURY, «Sovereignty and Inequality", European Journal of International Law, vol. 9, 1998, pp. 599 ss. 